\title{
Experiment Analysis of Cutting Temperature in Near Dry Machining of Stainless Steel AISI-202
}

\author{
Jagmeet Singh ${ }^{1}$, Gurpreet Singh ${ }^{2}$ \\ ${ }^{1,2}$ A. P, Department of Mechanical Engineering \\ Chandigarh University Gharuan, Mohali, India \\ ${ }^{1}$ er.jagmeetdhaliwal@gmail.com, ${ }^{2}$ gssingh410@gmail.com
}

\begin{abstract}
The tool wear produced in metal cutting operations due to high friction at tool work interface, which affect the tool life, product quality. For reducing the friction at tool work interface the various lubricants are used for cooling and lubrication. But Cooling and lubrication of work piece is dependent upon type of lubricant and lubrication methods used. The different Cooling and lubrication are Conventional Flood lubrication, near dry machining, Cryogenic cooling and cooling by Nano particle mixed with different oils. But Machining in current scenario is different from few years ago, because of high productivity, accuracy and environment. So it is necessity of present day of machining to work with technology which would not affect the environment and give the better quality of products. As already been mentioned that the cooling and lubrication ability of any technique depends upon the type of lubricant but there is lack of study on the distance of cooling nozzle from the cutting zone, angle of nozzle during turning operation. So therefore the effect of varying the nozzle distance on cutting temperature. The experimentation has been conducted by turning of A1S1202 stainless steel with Dry, Flood and Near dry machining with vegetable based oil. The temperature has been measured by $K$ thermocouple. The vegetable oil has been selected because of its environmental friendly conduct and does not produce health endanger, like mineral oil at some stage in flood lubrication.The present investigations show that there is Considerable variation in cutting temperature when nozzle distance and angle is varied. NDM in present study has reduced the Cutting temperature by 30 to $50 \%$.
\end{abstract}

Index Terms-NDM, HSS, DOC, RPM, MV, MM, M/MIN 